\title{
Pressure Ulcer Wound Care for Elderly in Home: A Case Report
}

\author{
Shuk-Fan Tong, Joanne Yip*, Kit-Lun Yick and Marcus Chun-Wah Yuen \\ Institute of Textiles and Clothing, The Hong Kong Polytechnic University, Hong Kong, China
}

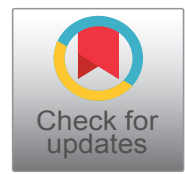

${ }^{*}$ Corresponding author: Joanne Yip, Institute of Textiles and Clothing, The Hong Kong Polytechnic University, Hung Hom, Hong Kong, China, E-mail: tcjyip@polyu.edu.hk

\begin{abstract}
Pressure ulcers are a frequent occurrence in the bedridden elderly, especially those who are over 65 years old. The healing of pressure ulcers is particularly difficult for these individuals as most of them may have different kinds of chronic illnesses and a low metabolism. Also, the healing process is complicated as there are numerous extrinsic and intrinsic contributing factors that influence the development and healing of pressure ulcers. Therefore, in this study, the healing progress of an eighty-six year old elderly individual with a Stage III heel ulcer is examined two months after he was discharged from the hospital. Along with regular follow-up consultations at the podiatry department in the hospital, proper wound care instructions are also given to the nursing home staff. It is found that careful and proper wound care, a sufficiently nutritious diet, regular repositioning and appropriate use of pressure ulcer preventive instruments are the main elements that enhance healing. The time required for healing may be a lengthy period, but still possible.
\end{abstract}

\section{Keywords}

Pressure ulcers, Heel ulcers, Elderly, Home for the elderly, Wound care

\section{Introduction}

Pressure sores, also commonly known as decubitus ulcers, pressure ulcers or bedsores, are a prevalent type of chronic wound among elderly, bedridden and wheelchair-bound patients, and individuals in long term care facilities [1,2]. The National Pressure Ulcer Advisory Panel (NPUAP) defines pressure ulcers as localized damage to the skin and underlying tissue $[2,3]$.

In general, the contributors of pressure ulcers can be divided into two types of factors: extrinsic and intrinsic factors. The former are the application of mechanical force on the skin and soft tissue over the bones while the latter are that which affect the skin architecture and the vascular and lymphatic system [4-8]. Extrinsic factors include shearing forces, pressure and friction, while intrinsic factors include age, nutrition, chronic illnesses, skin conditions and oxygen delivery $[5,9,10]$.

Shear stress is one of the major extrinsic factors of pressure sore development. The effect of the force of gravity on the human body means there will be contact force with the bed, wheelchair and other surfaces $[5,9,11]$. An inverse parabolic relationship between time and pressure was found in which higher shear stresses can induce pressure sores in a shorter period of time while lower shear stresses require a longer time to cause tissue damage [5]. Previous work in the literature has pointed out that a normal capillary filling pressure is around 32 and $12 \mathrm{mmHg}$ at the arteriolar and venous ends of the capillary respectively. Therefore, if the external pressure is greater than $32 \mathrm{mmHg}$, blood flow might be restricted to that particular area [6-8,12-14]. Apart from gravity force, shearing force and friction are also contributors to pressure ulcer development. Shearing is a mechanical force that originates from parallel loads. Consequently, the bones slide against resistance between the skin and its contact surface $[5,9,14]$. Besides, friction is created between the skin and contact surface which are moving across each other and thus leads to the initial breakdown of the skin. Moisture, which may come from urine, feces, perspiration and drainage from wounds, is also regarded as one of the extrinsic factors, as prolonged exposure may cause epidermis maceration and render the skin susceptible to injury $[5,9,14]$.

In terms of the intrinsic factors, pressure ulcers often occur in those who have impaired mobility or are seriously ill, and particularly affect older people as their rate of metabolism is reduced and skin becomes more delicate $[2,15]$. Malnutrition is also one of the critical contributors to pressure sore development. Researchers have proven that insufficient protein and energy intake are independent risk factors. In addition, the deficiency of vitamin $\mathrm{C}$ or minerals such as zinc and other trace elements may lead to the breakdown of tissues [5]. Moreover, people who have arterial disease, hypotension and diabetes are at high risk $[5,12,16]$. Previous research has identified that increasing skin and body temperatures contribute to increased perspiration and increase oxygen demand, thus making the skin more susceptible to erosion and infection. Also, dry and scaling skin reduces the resistance of tissue to mechanical forces [5]. On the other hand, the chances that pressure ulcers will form on moist skin are greater than on dry skin, as indicated by the literature. The maceration of the epidermis easily causes disintegration of the skin and tissue sloughs, and there is an increase in friction [14].

The reoccurrence of pressure ulcers can be frequent, and pressure ulcers are difficult to heal once they develop, especially in the elderly who are immobilized $[2,10,17]$. Previous researchers have proven that the rate of complete healing of pressure ulcers is as low as $10 \%$ and less than $13 \%$ of those in acute hospital settings heal in two weeks [18]. Researchers have also reported that during a time period of 6 months of healing, $72 \%$ of Stage II ulcers will heal in the elderly, while $45.2 \%$ and $30.6 \%$ of those with Stages III and V pressure ulcers 
will heal at the sixth month mark [19]. Previous research has also proven that pressure ulcers are the third most costly health disorder, following cancer and cardiovascular diseases [20]. Not only do ulcers affect the quality of life and result in longer hospitalizations, but they also constitute as a burden to families and caretakers, and cause multiple and life threatening medical complications $[1,3,17,18,20$ 22].

Researchers have reported that the incidence of pressure sores in the elderly is $10-25 \%$ and about $70 \%$ of them are older than 65 years old $[2,17,18,23]$, while nearly $57 \%$ of adult patients with a principal diagnosis of pressure ulcers are older than 65 years old [1]. For the hospitalized elderly and those in outpatient settings, the percentage incidence of pressure sores is $8.8 \%$ and $1.61 \%$ respectively, and $25.16 \%$ in Hong Kong nursing homes [23,24]. Previous researchers have proven that two thirds of pressure ulcers occur in patients who are over 70 years old and the prevalence is $17-28 \%$ for those in nursing homes [17]. There is also consensus that pressure ulcers are common in bedridden elderly patients who are in the community, nursing homes or hospitals. The prevalence of pressure ulcers in general is $4-30 \%$ for those in hospitals, $2.4-23 \%$ for those in long term care facilities and $4 \%$ for in home care patients. Previous work has also provided evidence that the prevalence of pressure ulcers has recently doubled while the percentage incidence of pressure ulcers has increased 80\% from 1995 to 2008 [19]. Researchers have reported that 159,000 nursing home residents (11\%) in the US had a pressure ulcer in 2004 [25]. Based on study results in Sweden, 97.7\% of the patients who are over the age of 65 years old had a pressure ulcer [19]. The percentage of those who are over 65 years old with a preexisting pressure ulcer at the time of hospital entry from nursing homes and other living situations is $26.2 \%$ and $4.8 \%$ respectively [26]. One fourth of all pressure ulcers in hospitalized patients begin in the operating room during surgery and more than $80 \%$ of hospitalized patients with pressure ulcers developed them in the first five days of their hospitalization [17]. In fact, pressure ulcers can occur a few hours after immobility induced pressure and very often near the end of the life of patients $[3,22]$.

In order to prevent and treat pressure ulcers, different methods are adopted to determine the appropriate prevention interventions including facility-specific standardized protocols, individualized care plans and orders from physicians or nurses [27]. The NPUAP in the US has also published a reference guide for pressure ulcer prevention and treatment, while Canada has its own practice guidelines for clinicians [28,29]. In Hong Kong, the Hong Kong Association of Gerontology as well as the Social Welfare Department has also provided some guidelines for nursing homes on pressure ulcer prevention and treatment [30,31]. In general, the management of pressure ulcers focuses on avoiding further deterioration of the ulcers by regular repositioning and the use of pressure relief devices, maintaining cleanliness of the wound and balancing the level of moisture of the ulcer, thus preventing the development of infections and minimizing the pain of patients [32].

Smith reviewed the literature on the treatment of pressure ulcers in nursing homes while Versluysen investigated the prevalence of pressure ulcers in the elderly with orthopedic problems $[4,33,34]$.

Schubert examined the effects of phototherapy on the healing process of pressure ulcers in elderly patients [34]. Also, Brem et al. tracked the healing of elderly patients with pressure ulcers between the ages of 65 to 102 but did not record detailed information about the treatment provided [35]. Liu and Dai studied the influence of medical and nutritional approaches on the healing of pressure ulcers in the elderly while Cereda et al. investigated the differences between using a disease-specific approach and standard dietary approach for pressure ulcer healing in the elderly $[23,36]$. Berlowitz et al. investigated whether the quality of nursing home care is improving in terms of treating pressure ulcers [37]. Baumgarten et al. also reported incidences of pressure ulcers in the elderly during the first two days of hospital stay and the characteristics of patients which may increase the likelihood of pressure development [22]. Belmin et al. and Hopkins et al. examined the effects of applying negative pressure wound therapy for Stage IV pressure ulcers by using different types of wound dressings in the elderly [38-40]. The latter studied the experience of elderly individuals with pressure ulcers [40].

Pressure ulcer wound healing is complicated and related to different extrinsic and intrinsic factors. Although there is numerous research work that investigates the performance of pressure ulcer preventive devices and treatments $[5,7,11,18,41,42]$, there is still a lack of information or studies on the wound healing progress of heel pressure ulcers in the elderly especially of those in regular nursing homes. Therefore, this study will report on a patient who is living at a home for the elderly with a Stage III heel pressure ulcer, and treated through normal wound care practices by using thick gauze instead of specially designed wound dressings. This study is important as it provides detailed information on real life situations; that is, the type of treatment adopted in nursing homes and the healing progress of Stage III heel pressure ulcers in the elderly, by considering different intrinsic and extrinsic factors.

\section{Case Report}

An eighty-six year old bedridden elderly man with diabetes, hypertension, benign prostatic hyperplasia, heart disease, cataracts, syphilis, history of duodenal ulcers and a right femoral fracture was placed into a local home for the elderly in Hong Kong. With large intestinal hemorrhage due to intestinal cancer, he was admitted to the hospital and underwent colostomy surgery. After surgery, he was placed in the rehabilitation department for recuperation. After approximately one and half months, he was discharged from the hospital but with a Stage III ischemic heel ulcer on his left foot. Initially, the area of his pressure ulcer was $6 \mathrm{~cm} \times 8 \mathrm{~cm}$.

The patient was required to undergo regular follow-up consultations at the podiatry department in the hospital. Instructions for the wound treatment were given by the podiatry department to the nursing home staff after each consultation. For the first twelve days, aseptic technique and iodosorb paste were applied on the wound base with a thick gauze every day. All of the applied iodosorb paste on the wound base was thoroughly removed by using normal saline water during cleansing and both sides of the white carrier gauze was to be removed before putting on a new application. From the thirteenth day onwards, normal saline water was used to remove the applied iodosorb paste first and then an aqueous betadine solution was applied for disinfection purposes. After cleansing, thick gauze along with betadine ointment was applied on the wound base once daily and held with surgical tape. Also, thick padded heel protectors were applied both during the day and at night, and the left posterior heel was allowed to be slightly elevated off the mattress by using a low pillow. Our first visit started two months after his discharge from the hospital and continued every week to record his healing progress. In the second follow-up consultation, a professional in the podiatry department at the hospital gave instructions to the nursing home staff for the wound care treatment. The instructions were that the betadine solution would continue to be applied to the heel ulcer and normal saline water was eliminated. After that, thick gauze with betadine ointment was applied onto the wound base once daily as previously done.

\section{Discussion}

The bedridden elderly patient in our study underwent colostomy surgery at a public hospital and stayed in the hospital for nearly one and half months. During his stay, he developed a pressure ulcer on his heel. He has different chronic illnesses, such as diabetes, hypertension, benign prostatic hyperplasia, and heart disease. Based on previous literature on intrinsic factors, he is regarded to have high risk for pressure ulcer development. Also, as he underwent colostomy surgery, his appetite was poor which severely affected his nutritional intake. In addition, his mobility was impaired and his body had not recovered from the surgery. Therefore, he was not able to turn by himself. However, insufficient pressure relief of the bony prominent parts of his body would then likely lead to pressure ulcer development. 


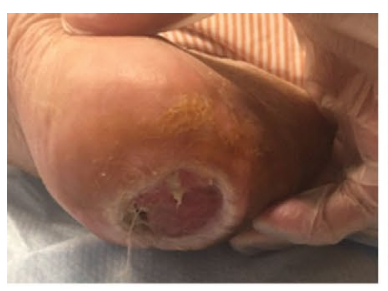

First visit

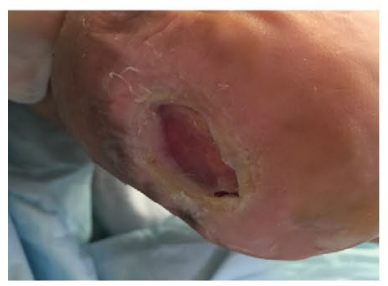

Fourth visit

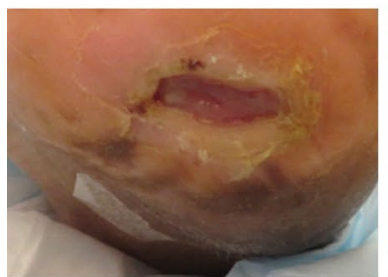

Seventh visit

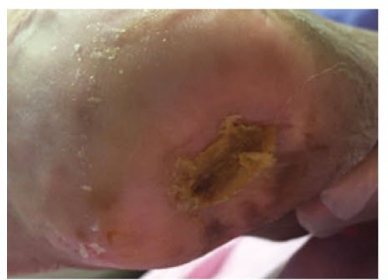

Tenth visit

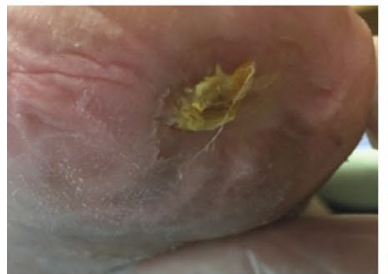

Thirteenth visit

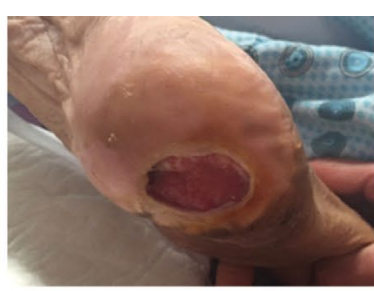

Second visit

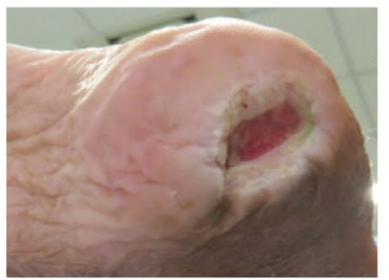

Fifth visit

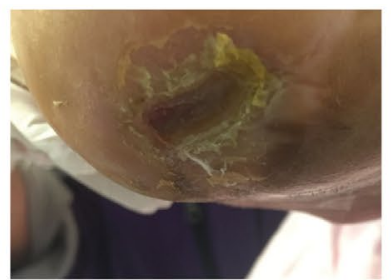

Eighth visit

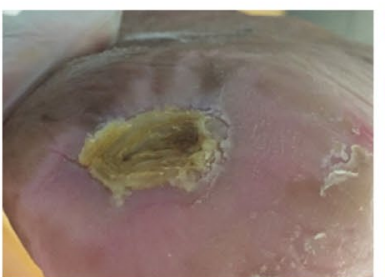

Eleventh visit

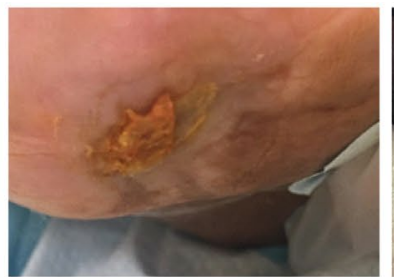

Fourteenth visit

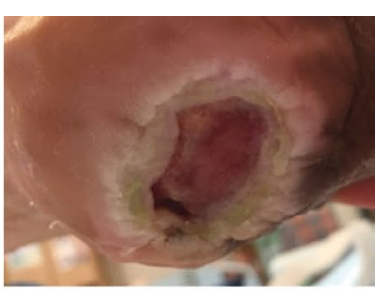

Third visit

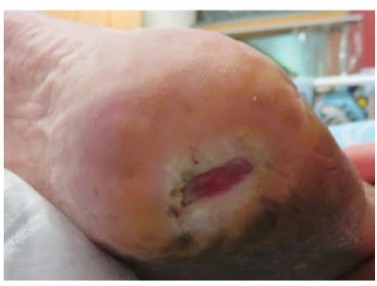

Sixth visit

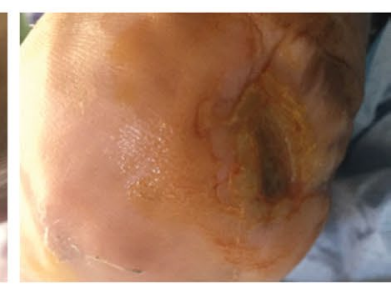

Ninth visit

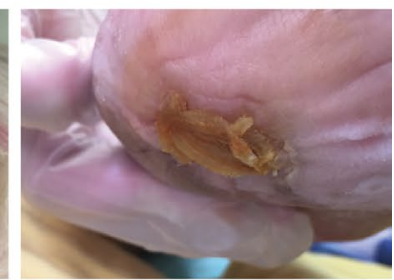

Twelfth visit

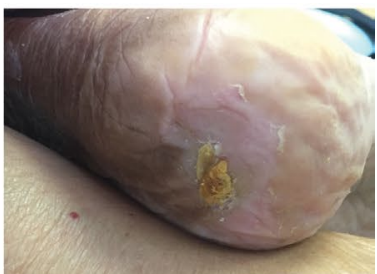

Fifteenth visit

Figure 1: The healing of the heel ulcer of this elderly individual continues to date.

At our first visit, the heel ulcer was highly exudative and the wound was large and deep. During the first four visits, there was a thick layer of soft white tissue around the wound base, which was red in color. The routine of the nursing home was that after bathing with the help of the staff in the morning, the heel ulcer wound was washed with a betadine solution and covered with thick gauze, and betadine ointment was applied onto the wound base. After applying the wound dressing, heel protectors and cushions were used and repositioning was carried out every two hours to avoid prolonged pressure onto the wound and other bony areas to prevent further development of the pressure ulcer. As the individual has diabetes and underwent colostomy surgery, he was given milk (mixed from a powder) to maintain his blood glucose level and received the required nutrition to facilitate the wound healing process. However, according to the observations of the nursing home staff, his appetite was poor after he was discharged from the hospital. Therefore, the healing progress of his heel ulcer was slower. On the fourth visit, he had a fever in the morning, and the nursing home staff sent him to a public hospital. He was subsequently hospitalized for one night. On the fifth visit, it became obvious that the size of the heel ulcer was becoming smaller and shallower. According to the staff, the amount of exudate was also reduced. Based on their observations, his appetite had also improved and he was able to ingest liquid food. On the seventh visit, a few days after the follow-up consultation, the thick white layer of soft tissue around the wound base had become harder, much like dead skin. There was a significant improvement in the heel ulcer wound on the eighth visit in which a layer of scab had developed which covered the wound base. Afterwards, the scab thickened and hardened, and the amount of exudate greatly decreased. The healing of the heel ulcer of this elderly individual continues to date ( Figure 1).

Although the elderly individual in this study has different chronic illnesses and is immobile with a low metabolism, his heel ulcer is gradually healing with progress due to proper wound care. According to an interview with the nursing home staff, a daily shower keeps his skin clean and improves his blood circulation so as to improve the wound healing process. Based on previous literature and reference guidelines published by the NPUAP, keeping skin clean by using warm water and a mild cleansing agent is an important skin care process that helps to minimize the irritation and dryness of the skin so as to prevent further ulceration and enhance wound healing $[28,43]$. The betadine solution and betadine ointment used have antibiotics 
which could have slowed down or stopped the growth of bacteria. Cleaning the wound with an antiseptic agent removes surface debris and remnants of dressing so as to prepare the wound bed for healing. In addition, the wound is covered with thick gauze in our case instead of special wound dressings that are designed for pressure ulcers. Not only does the thick gauze reduce the burden of the ulcer on the elderly individual and his family, but it is also good for absorption of the exudates from the wound and provides a ventilated environment for wound healing.

It is well known that minimizing the mechanical load is regarded as one of the most critical preventive measures to enhance wound healing as mechanical forces may cause further ulceration. Therefore, in our study, different pressure relief strategies including regular repositioning every two hours, the use of a pressure relieving mattress, cushions and heel protectors, and elevating the heel are also adopted to redistribute the pressure on the heel and avoid contact of the ulcer wound with the mattress so as to reduce the impacts of the pressure ulcer wound and occurrence of further ulcerations.

Proper nutrition is also one of the most important factors that enhances pressure ulcer wound healing. Researchers have indicated that patients with a higher protein intake experience more rapid healing of pressure ulcers as opposed to those with insufficient caloric intake of protein $[36,43,44]$. Also, Liu and Dai proved that malnutrition increases the severity of wound infections, and reduces the deposition of collagen and the tensile strength during healing [23]. Our study result demonstrates that the dietary intake greatly affects the wound healing process. Thus, the healing process is gradually enhanced when elderly individuals have better nutrition, especially in terms of more protein.

Based on the healing progress of the elderly individual here, it was found that different extrinsic and intrinsic factors of pressure ulcers should be avoided or enhanced by adopting appropriate treatment and care. Therefore, our result supports the contribution of pressure, nutrition and skin conditions to the healing of pressure ulcers.

\section{Conclusion}

A Stage III heel ulcer had developed in an immobile elderly man during his hospitalization after surgery. With a low metabolism and different kinds of chronic illnesses, more than six months is required to heal his heel ulcer. The healing process is long and complicated as many different contributing factors may affect the healing. However, due to the efforts of his caretakers, it is found that proper wound care, nutrition and daily care contribute to the healing of the ulcer wound, which is entirely possible, even if the individual is an elderly person.

\section{Acknowledgement}

Funding support for this investigation was provided by the Central Research Grant (G-YBD2) and a research studentship from The Hong Kong Polytechnic University which was granted to Tong Shuk Fan (RTNH).

\section{References}

1. Russo CA, Steiner C, Spector W (2008) Hospitalizations related to pressure ulcers among adults 18 years and older, 2006. Healthcare Cost and Utilization Project.

2. Tong SF, Yip J, Yick KL, Yuen MC (2016) Effects of different heel angles in sleep mode on heel interface pressure in the elderly. Clin Biomech 32: 229-235.

3. Margolis DJ, Bilker W, Knauss J, Baumgarten M, Strom BL (2002) The incidence and prevalence of pressure ulcers among elderly patients in general medical practice. Ann Epidemiol 12: 321-325.

4. Versluysen M (1985) Pressure sores in elderly patients. The epidemiology related to hip operations. J Bone Joint Surg Br 67: 10-13.

5. Murray LD, Magazinovic N, Stacey MC (2001) Clinical practice guidelines for the prediction and prevention of pressure ulcers. Primary Intention 9: 88-97.

6. Myers BA (2004) Wound management: principles and practice. ( $3^{\text {rd }}$ edn), Prentice Hall, New Jersey NJ.

7. Jaul E (2010) Assessment and management of pressure ulcers in the elderly current strategies. Drugs Aging 27: 311-325.
8. Somerset M (2007) Wound care fundamentals. Wound Care made incredibly easy. ( $2^{\text {nd }}$ edn), Lippincott Williams \& Wilkins, Philadelphia, PA, 51-70.

9. Bass MJ, Phillips LG (2007) Pressure sores. Curr Probl Surg 44: 101-143.

10. Bergstrom N, Braden B (1992) A prospective study of pressure sore risk among institutionalized elderly. J Am Geriatr Soc 40: 747-758.

11. Ostadabbas S, Yousefi R, Faezipour M, Nourani M, Pompeo M (2011) Pressure ulcer prevention: An efficient turning schedule for bed-bound patients. In Life Science Systems and Applications Workshop (LiSSA), IEEE/ $\mathrm{NIH}$ 159-162.

12. Tymec AC, Pieper B, Vollman K (1997) A comparison of two pressurerelieving devices on the prevention of heel pressure ulcers. Adv Wound Care 10: $39-44$.

13. Davies A, Williams JT (2009) The Use of spacer fabrics for absorbent medical applications. Journal of Fibre Bioengineering and Informatics 1: 321-330.

14. Maklebust J, Sieggreen M (2001) Pressure ulcers: Guidelines for prevention and management. ( $3^{\text {rd }}$ edn), Lippincott Williams \& Wilkins

15. Reenalda J, Jannink M, Nederhand M, IJzerman M (2009) Clinical use of interface pressure to predict pressure ulcer development: a systematic review. Assist Technol 21: 76-85.

16. http://www.webmd.com/skin-problems-and-treatments/four-stages-ofpressure-sores.

17. Landi F, Onder G, Russo A, Bernabei R (2007) Pressure ulcer and mortality in frail elderly people living in community. Arch Gerontol Geriatr 44: 217-223.

18. Thomas DR (2001) Prevention and treatment of pressure ulcers: what works? what doesn't? Cleve Clin J Med 68: 704-707

19. Jaul E, Menzel J (2014) Pressure Ulcers in the Elderly, as a Public Health Problem. Journal of General Practice 2: 174.

20. Schoonhoven L, Bousema MT, Buskens E (2007) The prevalence and incidence of pressure ulcers in hospitalized patients in the Netherlands: a prospective inception cohort study. Int J Nurs Stud 44: 927-935.

21. Pearson A, Francis K, Hodgkinson B, Curry G (2000) Prevalence and treatment of pressure ulcers in northern New South Wales. Aust J Rural Health 8: 103-110.

22. Baumgarten M, Margolis DJ, Localio AR, Kagan SH, Lowe RA, et al. (2006) Pressure ulcers among elderly patients early in the hospital stay. J Gerontol A Biol Sci Med Sci 61: 749-754.

23. Liu K, Dai L (2011) An Aggressive Medical-Nutritional Approach to the Management of Refractory Pressure Sores. Medical Bulletin 16.

24. Russo CA, Elixhauser A (2006) Hospitalizations related to pressure sores, 2003.

25. Park-Lee E, Caffrey $C$ (2009) Pressure ulcers among nursing home residents: United States, 2004. NCHS data brief 14: 1-8.

26. Keelaghan E, Margolis D, Zhan M, Baumgarten M (2008) Prevalence of pressure ulcers on hospital admission among nursing home residents transferred to the hospital. Wound Repair Regen 16: 331-336.

27. Wipke-Tevis DD, Williams DA, Rantz MJ, Popejoy LL, Madsen RW, et al. (2004) Nursing home quality and pressure ulcer prevention and management practices. J Am Geriatr Soc 52: 583-588.

28. http://www.npuap.org/wp-content/uploads/2014/08/Updated-10-16-14Quick-Reference-Guide-DIGITAL-NPUAP-EPUAP-PPPIA-160ct2014pdf.

29. Houghton PE, Campbell K, CPG P (2013) Canadian best practice guidelines for the prevention and management of pressure ulcers in people with Spinal Cord Injury: a resource handbook for clinicians. Ontario Neurotrauma Foundation.

30. http://www.hkag.org/PSDAS_book/ppt/15pdf.

31. http://www.swd.gov.hk/doc/LORCHE/lorchelet_c162_pdf.

32. Lyder CH, Ayello EA (2008) Pressure ulcers: a patient safety issue.

33. Smith, DM (1995) Pressure ulcers in the nursing home. Ann Intern Med 123: 433-442.

34. Schubert V (2001) Effects of phototherapy on pressure ulcer healing in elderly patients after a falling trauma. A prospective, randomized, controlled study. Photodermatol Photoimmunol Photomed 17: 32-38.

35. Brem H, Tomic-Canic M, Tarnovskaya A, Ehrlich HP, Baskin-Bey E, et al. (2001) Healing of elderly patients with diabetic foot ulcers, venous stasis ulcers, and pressure ulcers. Surg Technol Int 11: 161-167.

36. Cereda E, Gini A, Pedrolli C, Vanotti A (2009) Disease-specific, versus standard, nutritional support for the treatment of pressure ulcers in institutionalized older adults: a randomized controlled trial. J Am Geriatr Soc 57: 1395-1402

37. Berlowitz DR, Bezerra HQ, Brandeis GH, Kader B, Anderson JJ (2000) Are we improving the quality of nursing home care: the case of pressure ulcers. $J$ Am Geriatr Soc 48: 59-62. 
38. Baynham SA, Kohlman P, Katner HP (1999) Treating stage IV pressure ulcers with negative pressure therapy: a case report. Ostomy Wound Manage 45: 28-32.

39. Belmin J, Meaume S, Rabus MT, Bohbot S (2002) Sequential treatment with calcium alginate dressings and hydrocolloid dressings accelerates pressure ulcer healing in older subjects: a multicenter randomized trial of sequential versus nonsequential treatment with hydrocolloid dressings alone. J Am Geriatr Soc 50: 269-274.

40. Hopkins A, Dealey C, Bale S, Defloor T, Worboys F (2006) Patient stories of living with a pressure ulcer. J Adv Nurs 56: 345-353.
41. Bou JETI, Lopez JR, Camanes G, Narvaez EH, Blanco JB, et al. (2009) Preventing pressure ulcers on the heel: a Canadian cost study. Dermatol Nurs 21: 268-272.

42. Masaki N, Sugama J, Okuwa M, Inagaki M, Matsuo J, et al. (2013) Heel blood flow during loading and off-loading in bedridden older adults with low and normal ankle-brachial pressure index: a quasi-experimental study. Biol Res Nurs 15: 285-291.

43. http://www.hopkinsmedicine.org/geriatric_medicine_gerontology/_ downloads/readings/section8pdf.

44. Yoshikawa TT, Livesley NJ, Chow AW (2002) Infected pressure ulcers in elderly individuals. Clin Infect Dis 35: 1390-1396. 